\title{
Dissecting the cholera toxin-ganglioside GM1 interaction by isothermal titration calorimetry
}

\author{
W. Bruce Turnbull, Bernie L. Precious and Steve W. Homans*
}

\section{SUPPORTING INFORMATION}

\section{Synthesis of compounds 1-3}

Bovine brain gangliosides, Vibrio cholerae neuraminidase and bovine testes $\square$-galactosidase were purchased from Sigma. Leach ceramide glycanase was from Glyko. All other reagents were purchased from Sigma-Aldrich and were used without further purification. NMR spectra were acquired using either Varian Inova $\left({ }^{1} \mathrm{H} /{ }^{13} \mathrm{C} ; 500 / 125 \mathrm{MHz}\right)$ or Bruker Avance $250\left({ }^{1} \mathrm{H} /{ }^{13} \mathrm{C}\right.$; 250/62.5 MHz) systems. Spectra were referenced by standard methods. ${ }^{1}$ Electrospray mass spectra (ES-MS) were recorded using either a Micromass Platform II mass spectrometer in negative ion mode or a Micromass LCT (TOF) in positive ion mode. Optical rotations were recorded on an Optical Activity AA-1000 polarimeter at $22{ }^{\circ} \mathrm{C}$.

\section{Synthesis of GM1os and GM2os}

Mixed bovine brain gangliosides (100 mg) were treated with $V$. cholerae neuraminidase (8 U) in sodium acetate buffer $(50 \mathrm{mM}, \mathrm{pH} 5.5,10 \mathrm{ml})$ containing calcium chloride $(4 \mathrm{mM})$ at $37^{\circ} \mathrm{C}$ overnight. The product mixture was freeze dried and redissolved in water $(3 \mathrm{ml})$ before being purified by reversed phase chromatography on octyl sepharose $(1,90 \mathrm{~cm})$ eluting with a linear 
gradient of EtOH- $\mathrm{H}_{2} \mathrm{O}, 1: 1$ to EtOH to give ganglioside GM1 (42 mg). GM1 (15 mg) was then incubated for three days at $37^{\circ} \mathrm{C}$ in sodium acetate buffer $(10 \mathrm{mM}, \mathrm{pH} 5.0,1.4 \mathrm{ml})$ containing bovine serum albumin $(0.2 \mathrm{mg} / \mathrm{ml})$, Triton X100 $(4 \mathrm{mg} / \mathrm{ml})$ and ceramide glycanase $(3.12 \mathrm{U})$. The reaction mixture was extracted with $\mathrm{Et}_{2} \mathrm{O}$ and the aqueous layer was applied to a C-18 reversed phase silica gel column and the product, GM1os (1) $(10 \mathrm{mg})$, was eluted with $\mathrm{H}_{2} \mathrm{O}$. This material was further digested overnight at $37{ }^{\circ} \mathrm{C}$ with bovine testes $\square$-galactosidase $(0.5 \mathrm{U})$ in sodium acetate $(25 \mathrm{mM}, \mathrm{pH} 5.0,2 \mathrm{ml})$ containing bovine serum albumin $(2 \mathrm{mg} / \mathrm{ml})$. The protein was precipitated by heating at $100{ }^{\circ} \mathrm{C}$ for three minutes and removed by centrifugation. Following chromatography on Biogel P2 $\left(\mathrm{H}_{2} \mathrm{O}\right)$, the product GM2os (2) (4 mg) was freeze dried. No GM1os could be detected in this sample by either high performance anion exchange chromatography on a Dionex PA100 column (eluting with $100 \mathrm{mM} \mathrm{NaOAc/200} \mathrm{mM} \mathrm{NaOH)} \mathrm{or}$ electrospray mass spectrometry (ES-MS for compound 2: $\mathrm{m} / \mathrm{z}$ calcd for $\mathrm{C}_{31} \mathrm{H}_{51} \mathrm{~N}_{2} \mathrm{O}_{24}[\mathrm{M}-\mathrm{H}]^{-}$ 835.28, observed 835.30). ${ }^{1} \mathrm{H}$ and ${ }^{13} \mathrm{C}$ NMR spectra for compounds $\mathbf{1}$ and $\mathbf{2}$ were in agreement with those published previously. ${ }^{2,3}$

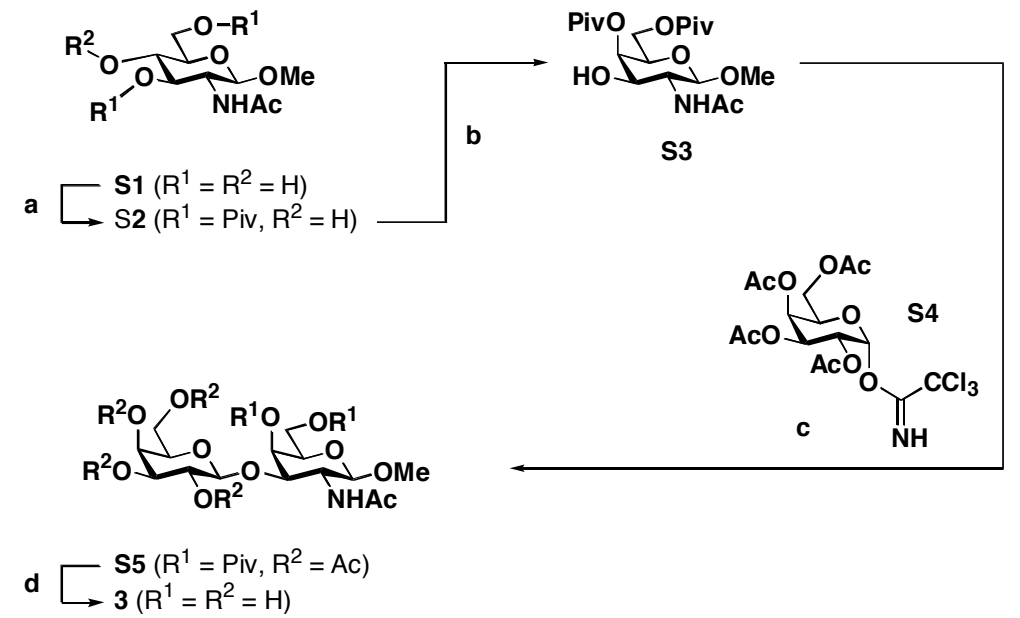

Scheme S1. Reagents: a) PivCl / $\mathrm{C}_{5} \mathrm{H}_{5} \mathrm{~N}$. b) (i) $\mathrm{Tf}_{2} \mathrm{O} / \mathrm{CH}_{2} \mathrm{Cl}_{2} / \mathrm{C}_{5} \mathrm{H}_{5} \mathrm{~N}$; (ii) $\mathrm{H}_{2} \mathrm{O} / \square$. c) S4 / $\mathrm{CH}_{2} \mathrm{Cl}_{2} / \mathrm{BF}_{3} \cdot \mathrm{OEt}_{2}$. d) $\mathrm{NaOMe} / \mathrm{MeOH}$. 


\section{Synthesis of methyl 2-acetamido-2-deoxy-3,6-di-O-pivaloyl-[-D-glucopyranoside}

Trimethylacetyl chloride (400 $\square 1,3.20 \mathrm{mmol}$ ) was added, dropwise, to a stirred solution of methyl 2-acetamido-2-deoxy- $\mathrm{C-D}$-glucopyranoside $\mathbf{S} \mathbf{1}^{4}$ (300 $\mathrm{mg}, 1.28 \mathrm{mmol}$ ) in dry pyridine (4.5 $\mathrm{ml})$ at room temperature. After two hours, the reaction was quenched by addition of $\mathrm{MeOH}(0.5$ $\mathrm{ml})$ and concentrated to an oil which was diluted with EtOAc $(50 \mathrm{ml})$ and washed sequentially with $\mathrm{HCl}(1 \mathrm{M})$, saturated $\mathrm{NaHCO}_{3}$ solution and saturated $\mathrm{NaCl}$ solution, before drying the organic extracts over $\mathrm{Na}_{2} \mathrm{SO}_{4}$. Following concentration, the crude product was subjected to column chromatography (silica gel; EtOAc- $\mathrm{PhCH}_{3}, 4: 1$ ) to give glucosyl diester $\mathbf{S} 2$ as an amorphous solid (300 mg, 58\%). [ [ $]_{\mathrm{D}}-33.5^{\circ}(c$ 1.0, $\mathrm{MeOH}) ;{ }^{1} \mathrm{H} \mathrm{NMR}\left(\mathrm{CDCl}_{3}, 250 \mathrm{MHz}\right)$ :

1.20, $1.23\left(2 \square \mathrm{s}, 18 \mathrm{H}, 2 \square \mathrm{CMe}_{3}\right), 1.94$ (s, 3H, NAc), 3.40 (brs, 1H, OH), 3.47 (s, 3H, OMe), 3.55 (m, 2H, H-4, H-5), 3.79 (m, 1H, H-2), 4.40 (m, 3H, H-1, H-6, H-6'), 4.92 (dd, $J_{2,3}=10.5, J_{3,4}$ $=8.5 \mathrm{~Hz}, 1 \mathrm{H}, \mathrm{H}-3), 6.03\left(\mathrm{~d}, J_{2, \mathrm{NH}}=9.4 \mathrm{~Hz}, 1 \mathrm{H}, \mathrm{NH}\right)$; ES-MS $m / z$ calcd for $\mathrm{C}_{19} \mathrm{H}_{33} \mathrm{NNaO}_{8}[\mathrm{M}+$ $\mathrm{Na}^{+}$426.2104, observed 426.2096 .

\section{Synthesis of methyl 2-acetamido-2-deoxy-4,6-di-O-pivaloyl-[-D-galactopyranoside}

Trifluoromethanesulfonic anhydride (190 $\square \mathrm{l}, 1.15 \mathrm{mmol})$ was added, dropwise, to a cooled (0 $\left.{ }^{\circ} \mathrm{C}\right)$ solution of diester $\mathbf{S 2}(350 \mathrm{mg}, 0.87 \mathrm{mmol})$ in $\mathrm{CH}_{2} \mathrm{Cl}_{2}(7 \mathrm{ml})$ and $\mathrm{C}_{5} \mathrm{H}_{5} \mathrm{~N}(350 \square \mathrm{l})$. After stirring for two hours at $0{ }^{\circ} \mathrm{C}$ and for a further two hours at room temperature, $\mathrm{H}_{2} \mathrm{O}(350 \square \mathrm{l}$ ) was added and the mixture was heated under reflux overnight. The mixture was then diluted with $\mathrm{CH}_{2} \mathrm{Cl}_{2}(50 \mathrm{ml})$ and washed sequentially with $\mathrm{HCl}(1 \mathrm{M})$, saturated $\mathrm{NaHCO}_{3}$ solution and saturated $\mathrm{NaCl}$ solution, before drying the organic extracts over $\mathrm{Na}_{2} \mathrm{SO}_{4}$. Following concentration, the crude product was subjected to column chromatography (silica gel; EtOAc- 
$\mathrm{MeOH}, 95: 5)$ to give galactosyl diester $\mathbf{S 3}$ as an amorphous solid (170 mg, 48\%). [D] $]_{\mathrm{D}}-17.8^{\circ}(c$ 1.0, $\mathrm{MeOH}) ;{ }^{1} \mathrm{H} \mathrm{NMR}\left(\mathrm{CDCl}_{3}, 500 \mathrm{MHz}\right): \square$ 1.19, $1.26\left(2 \square \mathrm{s}, 18 \mathrm{H}, 2 \square \mathrm{CMe}_{3}\right), 2.05(\mathrm{~s}, 3 \mathrm{H}$, NAc), 3.51 (s, 3H, OMe), $3.67(\mathrm{~m}, 1 \mathrm{H}, \mathrm{H}-2), 3.89\left(\mathrm{pt}, J_{5,6}=J_{5,6}=6.7 \mathrm{~Hz}, 1 \mathrm{H}, \mathrm{H}-5\right), 3.99(\mathrm{~m}, 1 \mathrm{H}$, H-3), $4.09\left(\mathrm{dd}, J_{5,6}=6.1 \mathrm{~Hz}, J_{6,6^{\prime}}=11.2 \mathrm{~Hz}, 1 \mathrm{H}, \mathrm{H}-6\right), 4.17\left(\mathrm{dd}, J_{5,6^{\circ}}=7.3 \mathrm{~Hz}, J_{6,6^{\prime}}=11.2 \mathrm{~Hz}, 1 \mathrm{H}\right.$, H-6'), $4.44\left(\mathrm{~d}, J_{1,2}=8.3 \mathrm{~Hz}, 1 \mathrm{H}, \mathrm{H}-1\right), 4.49$ (brd, $\left.J_{3, \mathrm{OH}}=2.9 \mathrm{~Hz}, 1 \mathrm{H}, \mathrm{OH}\right), 5.30\left(\mathrm{~d}, J_{3,4}=3.0 \mathrm{~Hz}\right.$, $1 \mathrm{H}, \mathrm{H}-4), 5.78\left(\mathrm{~d}, J_{2, \mathrm{NH}}=4.5 \mathrm{~Hz}, 1 \mathrm{H}, \mathrm{NH}\right) ;{ }^{13} \mathrm{C} \mathrm{NMR}\left(\mathrm{CDCl}_{3}, 62.5 \mathrm{MHz}\right): \square 23.8,27.5(3 \mathrm{C}), 27.6$ (3C), 39.1, 39.6, 55.7, 57.1, 62.3, 68.8, 71.7, 72.4, 101.7, 173.6, 178.3, 178.4; ES-MS $m / z$ calcd for $\mathrm{C}_{19} \mathrm{H}_{34} \mathrm{NO}_{8}[\mathrm{M}+\mathrm{H}]^{+}$404.2284, observed 404.2278.

\section{Synthesis of methyl 2,3,4,6-tetra-O-acetyl-[-D-galactopyranosyl-(1] 3)-2-acetamido-2-}

\section{deoxy-4,6-di- $O$-pivaloyl-C-D-galactopyranoside}

Acceptor S3 (100 mg, $0.25 \mathrm{mmol}$ ) was glycosylated with trichloroacetimidate $\mathbf{S 4}$ (240 mg, $0.50 \mathrm{mmol})$ and $\mathrm{BF}_{3} \cdot \mathrm{OEt}_{2}$ in dry $\mathrm{CH}_{2} \mathrm{Cl}_{2}(2 \mathrm{ml})$, heated under reflux for four hours. The reaction mixture was cooled to room temperature, neutralised with $\mathrm{Et}_{3} \mathrm{~N}$ and concentrated to an oil that was purified by column chromatography (silica gel; Hexane-EtOAc, 1:9, then EtOAc) to give disaccharide $\mathbf{S 5}$ as an amorphous solid $(120 \mathrm{mg}, 66 \%)$. [0] $]_{\mathrm{D}}+11.3^{\circ}(c 1.0, \mathrm{MeOH}) ;{ }^{1} \mathrm{H}$ NMR $\left(\mathrm{CDCl}_{3}, 500 \mathrm{MHz}\right): \square \quad 1.17,1.21\left(2 \square \mathrm{s}, 18 \mathrm{H}, 2 \square \mathrm{CMe}_{3}\right), 1.93,1.95,2.01,2.03,2.09(5 \square \mathrm{s}, 15 \mathrm{H}$, $5 \square \mathrm{Ac}$ ), $3.20(\mathrm{~m}, 1 \mathrm{H}, \mathrm{H}-2 \mathrm{a}), 3.46$ (s, 3H, OMe), 3.85 (m, 2H, H-5a, H-5b), 3.97 (dd, $J_{5,6}=8.0$ $\left.\mathrm{Hz}, J_{6,6^{\prime}}=11.4 \mathrm{~Hz}, 1 \mathrm{H}, \mathrm{H}-6 \mathrm{a}\right), 4.06\left(\mathrm{~m}, 2 \mathrm{H}, \mathrm{H}-6 \mathrm{~b}, \mathrm{H}-6 \mathrm{~b}^{\prime}\right), 4.12\left(\mathrm{dd}, J_{5,6^{\prime}}=4.7 \mathrm{~Hz}, J_{6,6^{\prime}}=11.4 \mathrm{~Hz}\right.$, 1H, H-6a'), 4.57 (d, $\left.J_{1,2}=7.8 \mathrm{~Hz}, 1 \mathrm{H}, \mathrm{H}-1 \mathrm{~b}\right), 4.67$ (dd, $\left.J_{2,3}=10.7 \mathrm{~Hz}, J_{3,4}=3.4 \mathrm{~Hz}, 1 \mathrm{H}, \mathrm{H}-3 \mathrm{a}\right)$, $4.90\left(\mathrm{~d}, J_{1,2}=8.3 \mathrm{~Hz}, 1 \mathrm{H}, \mathrm{H}-1 \mathrm{a}\right), 4.93\left(\mathrm{dd}, J_{2,3}=10.4 \mathrm{~Hz}, J_{3,4}=3.3 \mathrm{~Hz}, 1 \mathrm{H}, \mathrm{H}-3 \mathrm{~b}\right), 5.05\left(\mathrm{dd}, J_{1,2}=\right.$ $\left.7.8 \mathrm{~Hz}, J_{2,3}=10.4 \mathrm{~Hz}, 1 \mathrm{H}, \mathrm{H}-2 \mathrm{~b}\right), 5.30\left(\mathrm{~d}, J_{3,4}=3.3 \mathrm{~Hz}, 1 \mathrm{H}, \mathrm{H}-4 \mathrm{~b}\right), 5.37\left(\mathrm{~d}, J_{3,4}=3.4 \mathrm{~Hz}, 1 \mathrm{H}, \mathrm{H}-\right.$ 4a), $5.77\left(\mathrm{~d}, J_{2, \mathrm{NH}}=6.9 \mathrm{~Hz}, 1 \mathrm{H}, \mathrm{NH}\right) ;{ }^{13} \mathrm{C} \mathrm{NMR}\left(\mathrm{CDCl}_{3}, 62.5 \mathrm{MHz}\right): \square 20.9,21.0,21.1,21.2$, 
24.1, 27.4 (3C), 27.6 (3C), 39.1, 39.4, 55.9, 57.4, 61.4, 62.9, 67.2, 69.0, 69.5, 70.9, 71.2, 72.0, $75.5,100.3,101.4,169.5,170.5,170.6,170.8,171.2,177.2,178.2$; ES-MS $m / z$ calcd for $\mathrm{C}_{33} \mathrm{H}_{51} \mathrm{NNaO}_{17}[\mathrm{M}+\mathrm{Na}]^{+} 756.3055$, observed 756.3085 .

\section{Synthesis of methyl Q-D-galactopyranosyl-(1口 3)-2-acetamido-2-deoxy- $\square-D-$} galactopyranoside

Protected disaccharide $\mathbf{S 5}$ was de-esterified with NaOMe in $\mathrm{MeOH}$ (43 mM, $2 \mathrm{ml}$ ). After 24 hours at room temperature, a thick gel had formed which was dissolved on addition of water (2 $\mathrm{ml}$ ) and neutralised with $\mathrm{AcOH}$. The disaccharide was purified by gel filtration chromatography (Sephadex LH20; $\mathrm{H}_{2} \mathrm{O}$ ) to give the product 3 as an amorphous solid after freeze drying (40 mg, 58\%). [0] $]_{\mathrm{D}}+8.3^{\circ}\left(c\right.$ 1.0, $\left.\mathrm{H}_{2} \mathrm{O}\right) ;{ }^{1} \mathrm{H}$ NMR $\left(\mathrm{D}_{2} \mathrm{O}, 500 \mathrm{MHz}\right): \mathrm{Q} 2.05$ (s, 3H, Ac), 3.54-3.60 (m, 4H, H-2b, OMe), 3.65 (dd, $\left.J_{2,3}=9.1 \mathrm{~Hz}, J_{3,4}=3.2 \mathrm{~Hz}, 1 \mathrm{H}, \mathrm{H}-3 \mathrm{~b}\right), 3.68(\mathrm{~m}, 1 \mathrm{H}, \mathrm{H}-5 \mathrm{~b}), 3.74(\mathrm{~m}, 1 \mathrm{H}$, H-5a), 3.77-3.87 (m, 4H, H-6a, H-6a', H-6b, H-6b'), 3.90 (dd, $J_{2,3}=10.8 \mathrm{~Hz}, J_{3,4}=2.9 \mathrm{~Hz}, 1 \mathrm{H}$, H-3a), $3.94\left(\mathrm{~d}, J_{3,4}=3.2 \mathrm{~Hz}, 1 \mathrm{H}, \mathrm{H}-4 \mathrm{~b}\right), 4.04\left(\mathrm{dd}, J_{1,2}=8.5 \mathrm{~Hz}, J_{2,3}=10.8 \mathrm{~Hz}, 1 \mathrm{H}, \mathrm{H}-2 \mathrm{a}\right), 4.21(\mathrm{~d}$, $\left.J_{3,4}=2.9 \mathrm{~Hz}, 1 \mathrm{H}, \mathrm{H}-4 \mathrm{a}\right), 4.47\left(\mathrm{~d}, J_{1,2}=7.6 \mathrm{~Hz}, 1 \mathrm{H}, \mathrm{H}-1 \mathrm{~b}\right), 4.48\left(\mathrm{~d}, J_{1,2}=8.5 \mathrm{~Hz}, 1 \mathrm{H}, \mathrm{H}-1 \mathrm{a}\right) ;{ }^{13} \mathrm{C}$ NMR (HSQC, $\mathrm{D}_{2} \mathrm{O}, 125 \mathrm{MHz}$ ): $\square 21.9,50.7,56.5,60.5$ (2C), 67.7, 68.2, 70.0, 72.1, 74.3, 74.4, 79.4, 101.7, 104.2; ES-MS $m / z$ calcd for $\mathrm{C}_{15} \mathrm{H}_{27} \mathrm{NNaO}_{11}[\mathrm{M}+\mathrm{Na}]^{+} 420.1482$, observed 420.1468

\section{References}

(1) Gottlieb, H. E.; Kotlyar, V.; Nudelman, A. J. Org. Chem. 1997, 62, 7512-7515.

(2) Sillerud, L. O.; Yu, R. K.; Schafer, D. E. Biochemistry 1982, 21, 1260-1271.

(3) Sabesan, S.; Bock, K.; Lemieux, R. U. Can. J. Chem. 1984, 62, 1034-1045.

(4) Conchie, J.; Levvy, G. A. In Methods in Carbohydrate Chemistry; Whistler, R. L., Wolfram, M. L., BeMiller, J. N., Eds.; Academic Press Inc.: New York, 1963; Vol. II, pp 332335. 